\title{
Performance Comparison of Various Whiteness Formulas Based on Visual Evaluation Experiments
}

\author{
Ichiro KATAYAMA ${ }^{\dagger}$
}

Kindai University, Department of Human Factors Engineering and Environmental Design, Faculty of Biology-Oriented Science and Technology

Received September 2, 2018, Accepted April 26, 2019

\begin{abstract}
The Technical Committee TC 1-95 established in the International Commission on Illumination (CIE) is currently active in improving the CIE whiteness formula to extend the scope of the applicability of lighting conditions other than the standard illuminant D65. However, it is important to examine whether or not the $\mathrm{CIE}$ whiteness formula is significantly superior to other whiteness formulas under the standard illuminant D65. In this study, we conducted comparisons of the predictive performance of 19 whiteness formulas, including the CIE whiteness formula. These comparisons were based on the results of nine visual evaluation experiments that were conducted by four research groups. The results of the analysis clearly demonstrated that, compared to the CIE whiteness formula, the Uchida and Grum whiteness formulas had better outcomes. The predictive performance of the CIE whiteness formula was ranked 11th among the 19 whiteness formulas. This low predictive performance is due to the structure of the CIE whiteness formula, which does not have a penalty term. We also found that the structure of the Grum whiteness formula was simpler and had better predictive performance than the Uchida whiteness formula. Thus, we conclude that the Grum whiteness formula is superior to the Uchida whiteness formula.
\end{abstract}

KEYWORDS: whiteness formula, visual evaluation, perceived whiteness, predictive performance

\section{Introduction}

Quantitative evaluations of whiteness have been performed since around $1935^{1-3)}$. Activities aimed at establishing an international standard started in 1969, when the Subcommittee on Whiteness was established by the Technical Committee TC 1.3 of the International Commission on Illumination (CIE $)^{4}$. After some bumps and detours, the CIE recommended the CIE whiteness formula in $1986^{5}$. In 1987, the Committee on Specification Method of Whiteness (CSMW) was established by the Color Science Association of Japan and it examined if the CIE whiteness formula could be adopted as a Japanese Industrial Standard (JIS) ${ }^{6}$. As a result, the correlation between the results of visual evaluation experiments conducted by the committee and the CIE whiteness indices was low, and the range of acceptable white in Japan was assumed to be wider than the applicable range of the CIE whiteness formula. Although those problems were pointed out, to conform with international trends, the CIE whiteness formula was adopted as a JIS in $1991^{7}$.

The CIE whiteness formula, like many other whiteness formulas, is designed to predict the whiteness of a sample under the standard illuminant D65. Recently,
TC1-95, a technical committee aimed at extending the scope of the applicability of lighting conditions other than the standard illuminant D65, was established at the International Commission on Illumination, and the committee is now engaged in these activities ${ }^{8}$. However, it is important to first examine whether or not the current CIE whiteness formula is significantly superior to other whiteness formulas under the standard illuminant D65. Therefore, in this study, with the aim of providing useful evidence for the activities of TC1-95, we conducted comparisons of the predictive performance of 19 whiteness formulas, including the CIE whiteness formula. These comparisons were based on the results of nine visual evaluation experiments that were conducted by four research groups ${ }^{\text {?) }}$.

\section{Visual Evaluation Experiments}

Regarding the whiteness studies, data sets that fully contain the colorimetric values of the samples used for visual evaluations and the results corresponding to them are not publicly available; however, we obtained data sets for the visual evaluation experiments by the following research groups: (1) The CSMW in Japan, (2) Kurashiki City College (KCC) in Japan, (3) The Special

\footnotetext{
${ }^{\dagger}$ Corresponding Author: Ichiro Katayama katayama@waka.kindai.ac.jp
} 
Interest Group on Whiteness (SIGW) in Japan, and (4) Amirkabir University of Technology (AUT) in Iran.

The CSMW conducted the following three experiments in 1987 with the purpose of examining if the CIE whiteness formula should be adopted as a JIS as mentioned above. The evaluations included 18 types of white cloth samples by 74 observers (CSMW-I), 29 types of white paper samples by 74 observers (CSMW-II), and 47 samples with combined white cloth samples and white paper samples by 44 observers (CSMW-III). white cloth samples used in the experiments were unmercerised cotton cloth and polyester cloth that were whitened using fluorescent dye and bluing agent. The white paper samples were handmade paper with blue dye and fluorescent dye added to the pulp. The chromaticity scope of the samples was established to encompass a range with a high likelihood of being treated as white cloth or white paper within Japan. For the visual evaluation of whiteness, D65 fluorescent lamps were used as a light source, and the illuminance of the sample surface was about $1000 \mathrm{~lx}$. The sample presentation surface was N7 achromatic. The observers compared all the samples simultaneously and conducted a ranking of whiteness. The tristimulus values of each sample were calculated from the spectral radiance factor measured at $\mathrm{d}: 7^{\circ}$ geometry with a xenon lamp as a light source. The model of the spectrophotometer was not recorded.

The KCC conducted three evaluation experiments of polyester white cloth samples with 39 observers (KCCI, KCC-II, and KCC-III) in $2013^{10}$. In experiments KCC-I and KCC-II, 18 types of white cloth samples were used for evaluation, and in KCC-III, 17 samples were selected from the ones used in KCC-I and KCC-II. A light booth equipped with D65 fluorescent lamps was used. The sample presentation surface was N7 achromatic, and the illuminance of the sample surface was about $1500 \mathrm{~lx}$. The observers compared all the samples simultaneously and ranked the whiteness of the samples. A spectrophotometer (CMS-35SPX; Murakami Color Research Laboratory) was used to obtain the colorimetric values of the samples.

The SIGW, between 2006 and 2007, conducted an evaluation experiment (SIGW-2007) on 18 types of polyester white cloth samples with 69 observers at the Rochester Institute of Technology (RIT) in the United States, Joshibi University of Art and Design (JUAD) in Japan, and KCC in Japan ${ }^{11}$. In the evaluation experiment at the RIT, a Macbeth Spectra Light II light booth was used. The characteristic of the light source was set to Daylight+UV. The illuminance of the sample surface was about $1600 \mathrm{~lx}$. The experiment at the JUAD was performed under general illumination with D65 fluorescent lamp lighting. The sample presentation surface was N7 achromatic, and the illuminance of the sample surface was about $1200 \mathrm{~lx}$. In KCC, a light booth equipped with D65 fluorescent lamps was used. The sample presentation surface was N7 achromatic, and the illuminance of the sample surface was about $1500 \mathrm{~lx}$. Similarly, the observers compared all the samples simultaneously and ranked the whiteness of the samples. A spectrophotometer (CMS-35SPX; Murakami Color Research Laboratory) was used to obtain the colorimetric values of the samples. The observers in the experiments conducted at the RIT had various ethnic backgrounds, but it was confirmed that there was a very high correlation between the evaluation results at the RIT and the evaluation results by Japanese observers at the JUAD and $\mathrm{KCC}^{11)}$. From these results, we can assume that the observers' ethnic background does not have any influence when simply comparing whiteness. In addition, the SIGW conducted an evaluation experiment (SIGW-2014) on 12 types of near-white stimuli, which was presented on a liquid crystal display (LCD; CG223W; Eizo) in 2014 ${ }^{12)}$. Fifteen observers participated in the experiment. A white point on the LCD was adjusted to match the chromaticity of the standard illuminant D65. The hues of the presented stimuli were adjusted to 10PB, 3PB, 5B, 7BG, 9G, 3G, 3GY, 5Y, 4YR, 4R, 7P, and $\mathrm{N}$ in Munsell notation; the lightness was adjusted to 9.5 in Munsell notation; and the Munsell Chroma was set to 0.25 with the exception of the achromatic stimulus N. The background surrounding stimulus was N7 equivalent achromatic. The stimulus pair was displayed on the background for side-by-side comparison. The luminance of the white point on the LCD was $75.1 \mathrm{~cd} / \mathrm{m}^{2}$, whereas the luminance of both the stimulus and the background were 66.3 and $31.5 \mathrm{~cd} / \mathrm{m}^{2}$, respectively. A color luminance meter (CS-100; Minolta) was used to measure the $x y$ chromaticity coordinates and luminance.

Jafari and Amirshahi of the AUT conducted an evaluation experiment on 12 types of white cloth samples with 36 observers $^{13)}$. The samples consisted of cotton cloth that was scoured and bleached. A light booth (VeriVide) equipped with a D65 fluorescent lamp lighting was used. The observers evaluated the whiteness of the samples using the paired comparison method. It was stated that the colorimetric values of the samples were obtained using a spectrophotometer (Color-Eye 7000A; GretagMacbeth). However, only the $Y_{10}$ values were revealed publicly; hence, we read the chromaticity coordinates of the samples from Figure 1 of their $\operatorname{article}^{13)}$. When we compared the CIE whiteness indices calculated from the chromaticity coordinates that we read for each sample with the one published, we confirmed that they matched with $+0.1 \%$ to $-0.4 \%$ accuracy.

Figure 1 shows the distributions of $x_{10} y_{10}$ chromaticity and the $Y_{10}$ values of the samples used in the above-described visual evaluation experiments. The 

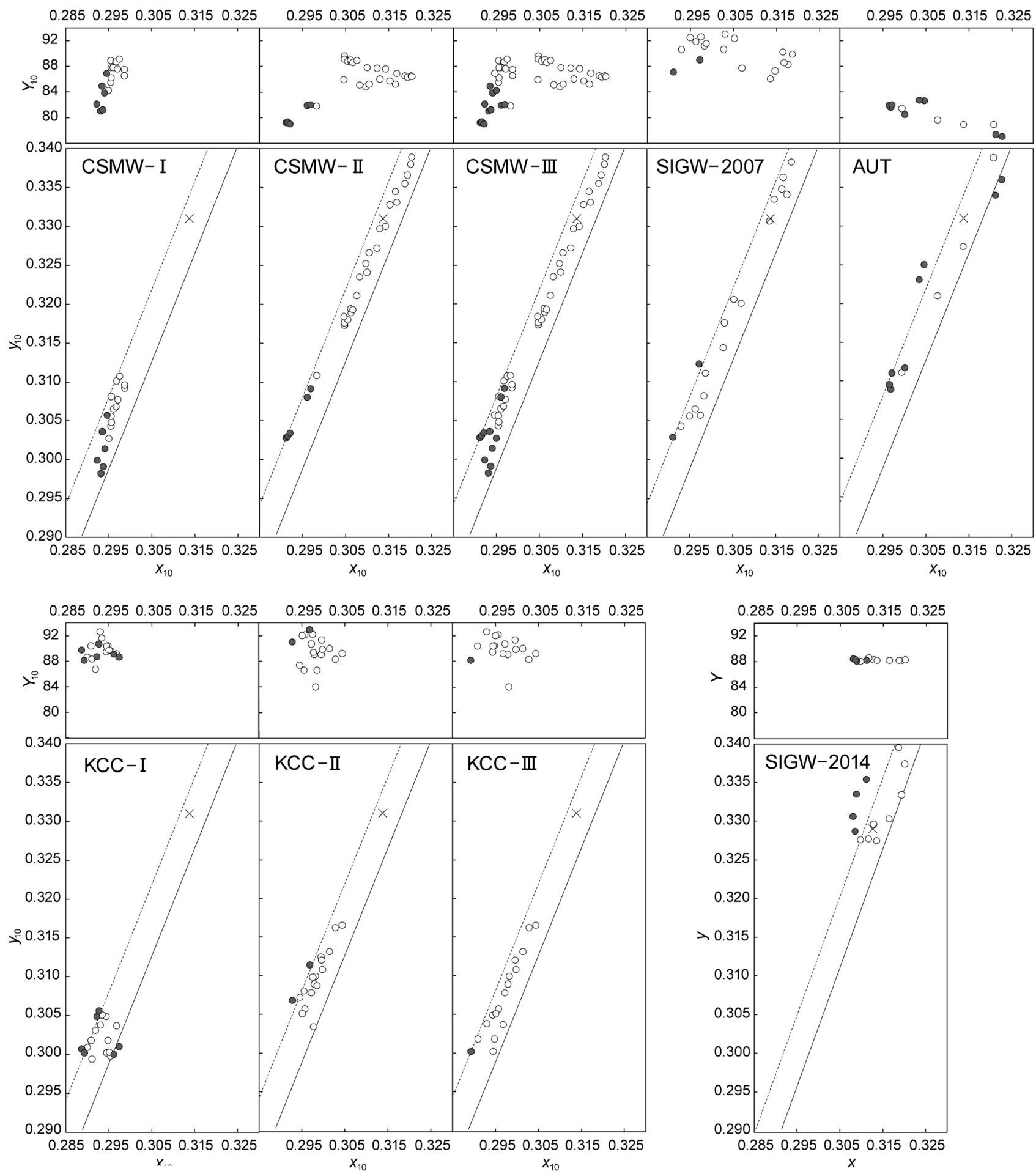

Figure 1 Distributions of $x_{10} y_{10}$ chromaticity and the $Y_{10}$ values of the samples used in the visual evaluation experiments.

open circles in the figure indicate the samples within the applicable scope of the CIE whiteness formula, and the filled circles indicate the samples outside the scope, respectively. The $\mathrm{x}$ marks indicate the chromaticity point of the standard illuminant D65. The dashed lines represent the boundary corresponding to the tint index, $T_{\mathrm{W}, 10}=2$, and the solid lines represent the boundary corresponding to the tint index, $T_{\mathrm{W}, 10}=-4$ (more details are described in Chapter 3). The colorimetric values of the SIGW-2014 experiment were calculated using a CIE 1931 standard colorimetric observer. In Figure 1, except for the SIGW-2014, it can be seen that the $Y_{10}$ values of the samples vary approximately in a range of the average value $\pm 3 \%$. A summary of the nine data sets is shown in Table 1.

In all the experiments mentioned above, the authors constructed interval scales of perceived whiteness based on the visual evaluation results; however, the 
Table 1 Summary of the nine data sets.

\begin{tabular}{|c|c|c|c|c|c|}
\hline Dataset ID & $\begin{array}{c}\text { Number of } \\
\text { samples }\end{array}$ & $\begin{array}{c}\text { Number of } \\
\text { observers }\end{array}$ & Evaluation method & $\begin{array}{c}\text { Level of } \\
\text { measurement }\end{array}$ & Material \\
\hline CSMW-I & 18 & 74 & Ranking & Interval scale & Cotton, polyester \\
\hline CSMW-II & 29 & 74 & Ranking & Interval scale & Paper \\
\hline CSMW-III & 47 & 44 & Ranking & Interval scale & Cotton, polyester, paper \\
\hline KCC-I & 18 & 39 & Ranking & Interval scale & Polyester \\
\hline KCC-II & 18 & 39 & Ranking & Interval scale & Polyester \\
\hline KCC-III & 17 & 39 & Ranking & Interval scale & Polyester \\
\hline SIGW-2007 & 18 & 69 & Ranking & Interval scale & Polyester \\
\hline SIGW-2014 & 12 & 15 & Paired comparison & Interval scale & Stimuli displayed on LCD \\
\hline AUT & 12 & 36 & Paired comparison & Ordinal scale & Cotton \\
\hline
\end{tabular}

AUT results did not meet the interval scale prerequisites and they achieved only ordinal scale values ${ }^{13}$. Furthermore, CIE whiteness only represents information as an ordinal scale ${ }^{5}$. Therefore, in the following analyses, we treated uniformly the visual evaluation results as the ordinal scale values.

\section{Whiteness Formulas Evaluated}

Whiteness formulas can be classified into the following five types depending on the structure of their formula ${ }^{14,15)}$ : (1) expressed by one of the tristimulus values, (2) expressed by the combination of two or three tristimulus values, (3) expressed as a function of lightness and chroma, (4) expressed as a color difference from the standard white point, and (5) other structures. We selected 16 typical whiteness formulas that fall under each type and in which whiteness index can be calculated based on CIEXYZ tristimulus values and three metrics considered to be related to the degree of whiteness. Thus, in total, we evaluated 19 whiteness formulas, including the CIE whiteness formula. Table 2 shows the whiteness formulas evaluated.

Most whiteness formulas classified as types 1 to 4 were proposed around 1960. Whiteness formulas classified as type 5 were proposed after the 1970s, and the CIE whiteness formula is included in this group.

Among the whiteness formulas shown in Table 2, only the CIE whiteness formula ${ }^{16)}$ explicitly stipulates its applicable range as shown in Equations (1) and (2):

$$
\begin{aligned}
& 40<W_{\mathrm{CIE}, 10}<5 Y_{10}-280,40<W_{\mathrm{CIE}}<5 Y-280 \\
& -4.0<T_{\mathrm{W}, 10}<2.0,-4.0<T_{\mathrm{W}}<2.0
\end{aligned}
$$

where $T_{\mathrm{W} .10}$ and $T_{\mathrm{W}}$ are tint indices, which are obtained from Equations (3) and (4), respectively:

$$
\begin{aligned}
& T_{\mathrm{W}, 10}=900\left(x_{\mathrm{n}, 10}-x_{10}\right)-650\left(y_{\mathrm{n}, 10}-y_{10}\right) \\
& T_{\mathrm{W}}=1000\left(x_{\mathrm{n}}-x\right)-650\left(y_{\mathrm{n}}-y\right)
\end{aligned}
$$

where $x_{10}, y_{10}$, and $x, y$ are the chromaticity coordinates of the sample, and $x_{\mathrm{n}, 10}, y_{\mathrm{n}, 10}$, and $x_{\mathrm{n}}, y_{\mathrm{n}}$ are the chromatic- ity coordinates of the perfect reflecting diffuser.

The CIE whiteness formula has not changed as it became a recommended standard in 1986, but in 2004 the range of the tint index $\left(T_{\mathrm{W}, 10}, T_{\mathrm{W}}\right)$ was changed as shown in Equation (2).

It has been reported that samples outside the applicable range of the CIE whiteness formula are also perceived as white ${ }^{6,26)}$. Thus, it should be noted that Equations (1) and (2) do not necessarily prescribe regions perceived as white in a CIEXYZ color space.

\section{Results of Analyses}

For each data set, we calculated the rank correlation coefficient between the predicted values obtained from the whiteness formulas and the visual evaluation results and then compared the predictive performance of each whiteness formula. The analysis procedure is shown in Figure 2, and the analysis results are shown in Table 3. The numbers shown in Figure 2 correspond to the numbers in Table 3. First, for each of the nine data sets, we calculated the Spearman's rank correlation coefficients between the predicted values by the 19 whiteness formulas and the visual evaluation results (1) in Table 3). Next, for each of the nine data sets, we calculated the order of the predictive performance of the 19 whiteness formulas (2) of Table 3). Through the above processing, each whiteness formula had nine rank values. Finally, based on the median of the nine rank values, we calculated the order of the predictive performance of the 19 whiteness formulas (3) of Table 3). The results showed that the predictive performances of $Y_{10}$, Hunter $L_{10}$, and $L_{10}^{*}$ were the best. The second best were the Grum whiteness formula ${ }^{\ddagger}$ and the Uchida whiteness formula. The CIE whiteness formula was ranked 11th among the 19 whiteness formulas. When we compared the worst case of the predictive performance rank among those whiteness formulas, each of the $Y_{10}$, Hunter $L_{10}, L_{10}^{*}$ and the CIE whiteness

\footnotetext{
\$ Rather than using the original formula, we used a simplified formula to calculate directly from the $x y$ chromaticity coordinates.
} 
Table 2 Whiteness formulas evaluated.

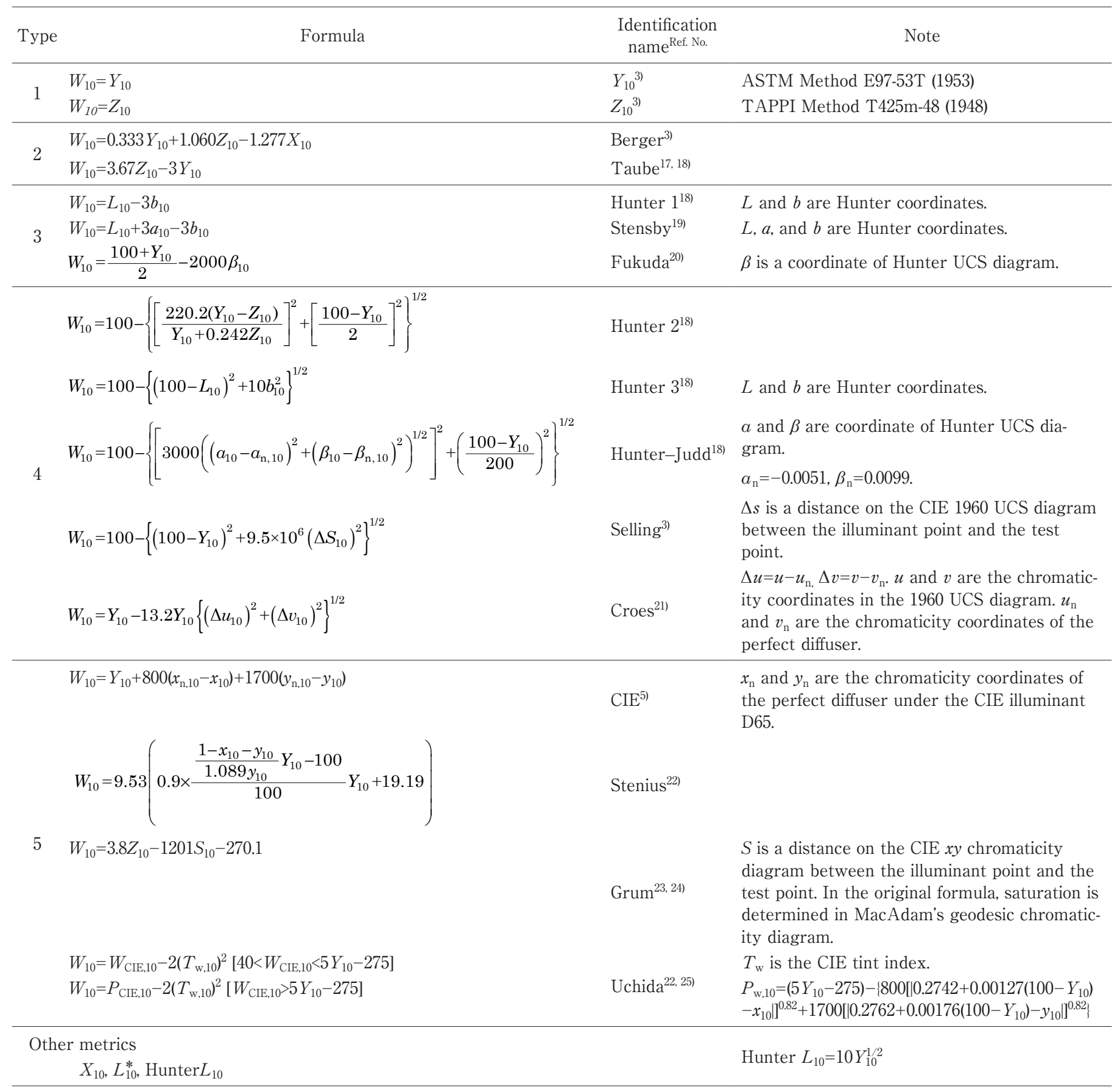

formulas was ranked 16th, the Uchida whiteness formula was ranked 13th, whereas the Grum whiteness formula was ranked 6 th. Figure 3 shows the predictive performance ranking of the $Y_{10}$, Hunter $L_{10}, L_{10}^{*}$, Grum, Uchida, and CIE whiteness formulas for each data set. In Figure 3, the abscissa represents the data set and the ordinate represents the ranking of the predictive performance. The figure also indicates that the Grum whiteness formula stably shows good predictive performance regardless of the data set.

$Y_{10}$, Hunter $L_{10}$, and $L_{10}^{*}$ had the best predictive performance; however, they cannot evaluate the influence of tint on the perceived whiteness by only one parameter of the lightness. Thus, in this study, we focused on the Grum and Uchida whiteness formulas, which had best predictive performances next to $Y_{10}$, Hunter $L_{10}$, and $L_{10}^{*}$, and then compared them to the CIE whiteness formula. Figure 4 shows the results. In the figure, the abscissa indicates each data set, and the ordinate denotes the Spearman's rank correlation coefficient. Our results showed that the predictive performance of the Uchida whiteness formula was significantly superior to that of the CIE whiteness formula in five data sets (CSMW-I, CSMW-III, KCC-I, KCC-III, and SIGW-2007). 
In addition, the predictive performance of the Grum whiteness formula was significantly better than that of the CIE whiteness formula in four data sets (CSMW-I, CSMW-II, CSMW-III, and KCC-II). The CIE whiteness formula did not show a significantly outstanding predictive performance in any of the data sets compared to the Uchida and Grum whiteness formulas. When we compared the Uchida whiteness formula to the Grum whiteness formula, the predictive performance of the latter was significantly excellent in three data sets (CSMW-I, CSMW-II, and AUT), whereas the former was significantly better in one data set only (KCC-I).

As all the data sets contained samples outside the scope of the CIE whiteness formula, we excluded those samples and conducted the same analysis. The results are shown in Figure 5. As only four samples in the AUT data set were within the scope of the CIE white-

Visually Evaluated Whiteness (9 Data Sets)

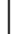

Predicted Whiteness (19 Whiteness Formulas)

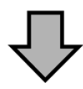

Spearman's Rank Correlation Coefficients

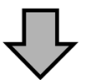

(1) $9 \times 19$ Correlation Matrix

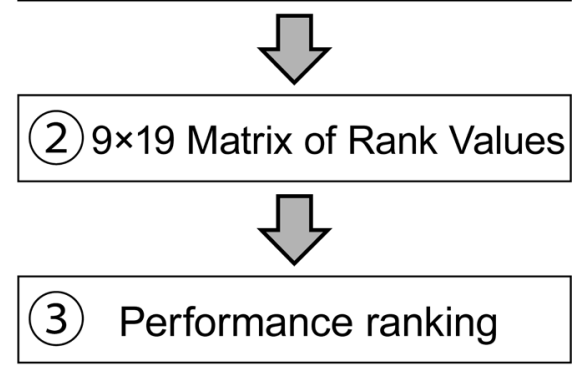

Figure 2 Analysis procedure for the performance comparison of whiteness formulas. ness formula, each of the CIE, Uchida, and Grum whiteness formulas had a rank correlation coefficient of 1.0; thus, we excluded the AUT data set from the analysis. In comparison to the CIE whiteness formula, the results showed that the Uchida whiteness formula had a significantly superior predictive performance in three data sets (KCC-I, KCC-III, and SIGW-2007) and also that the Grum whiteness formula had a significantly superior predictive performance in three data sets (CSMW-I, CSMW-II, and CSMW-III). The CIE whiteness formula did not show a significantly better predictive performance in any of the data sets in comparison to the Uchida and Grum whiteness formulas. When we compared the Uchida whiteness formula to the Grum whiteness formula, the predictive performance of the latter was significantly better in three data sets (CSMW-I, CSMWII, and CSMW-III). The Uchida whiteness formula did not show a significantly outstanding predictive performance in any of the data sets in comparison to the Grum whiteness formula.

Next, we examined why the predictive performance of the CIE whiteness formula is inferior to those of the Uchida and Grum whiteness formulas. Figure 6 shows the visual evaluation results of the nine data sets and the predicted values by the whiteness formulas in the order of CIE, Uchida, and Grum from the left column. The abscissa represents the $x_{10}$ value, the ordinate on the left represents the visual evaluation results, and the ordinate on the right represents the predicted values by the whiteness formulas. The blue dots indicate the visual evaluation results, and the red dots indicate the predicted values by the whiteness formulas. The $\mathrm{x}$ marks indicate the predicted values outside the applicable range of the CIE whiteness formula. We adjusted the scale on the ordinate on the right side so that each plot of the blue and red dots can overlap as much as possible. Figure 6 indicates that the predicted value by the CIE whiteness formula increases almost monotonously as the $x_{10}$ value decreases, i.e., as the bluish purity of the sample increases. However, in the CSMW-

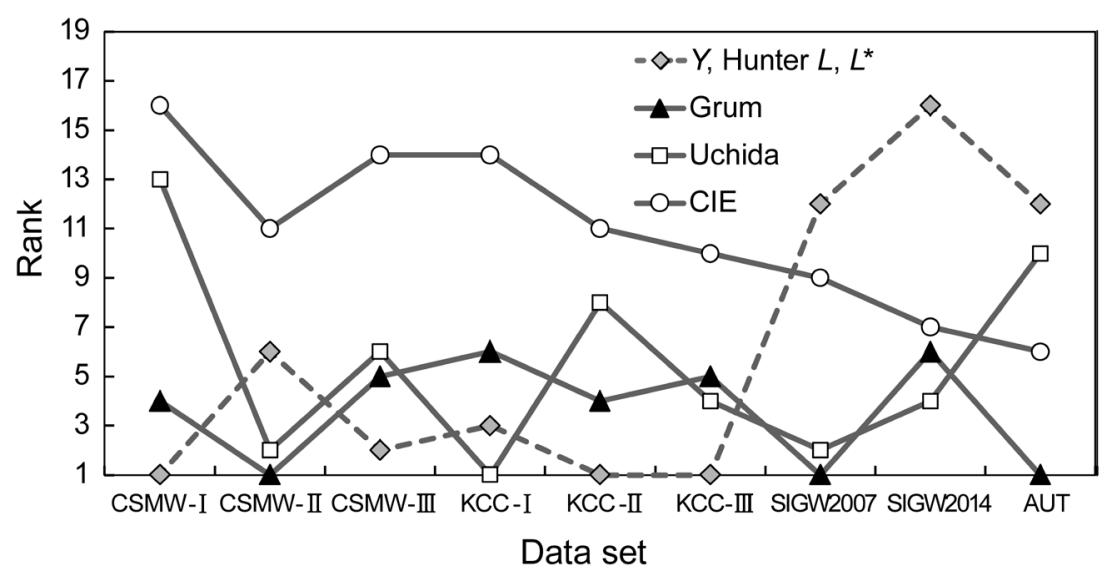

Figure 3 Predictive performance ranking of the $Y_{10}$, Hunter $L_{10}, L_{10}^{*}$, Grum, Uchida, and CIE whiteness formulas for each data set. 


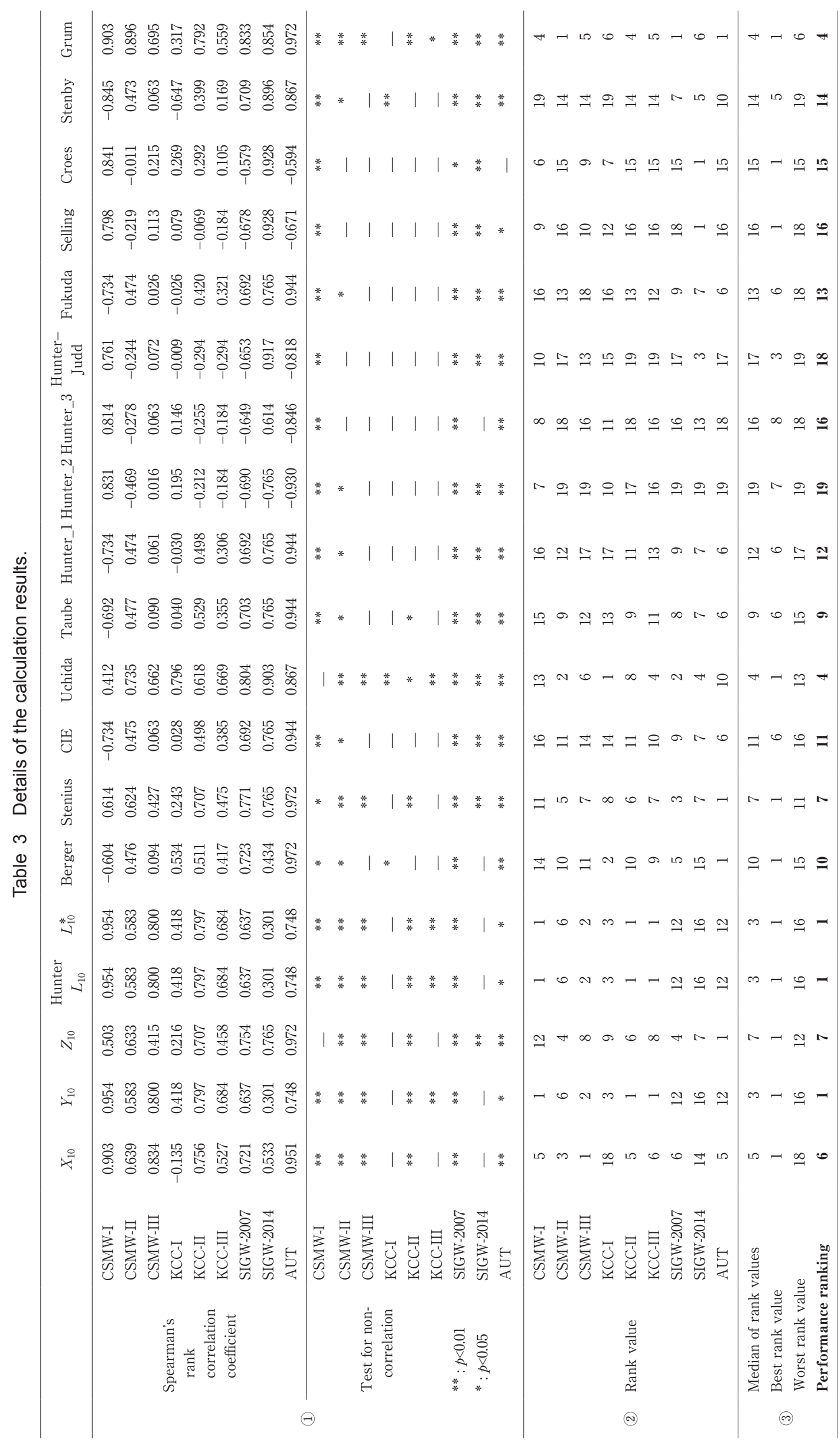



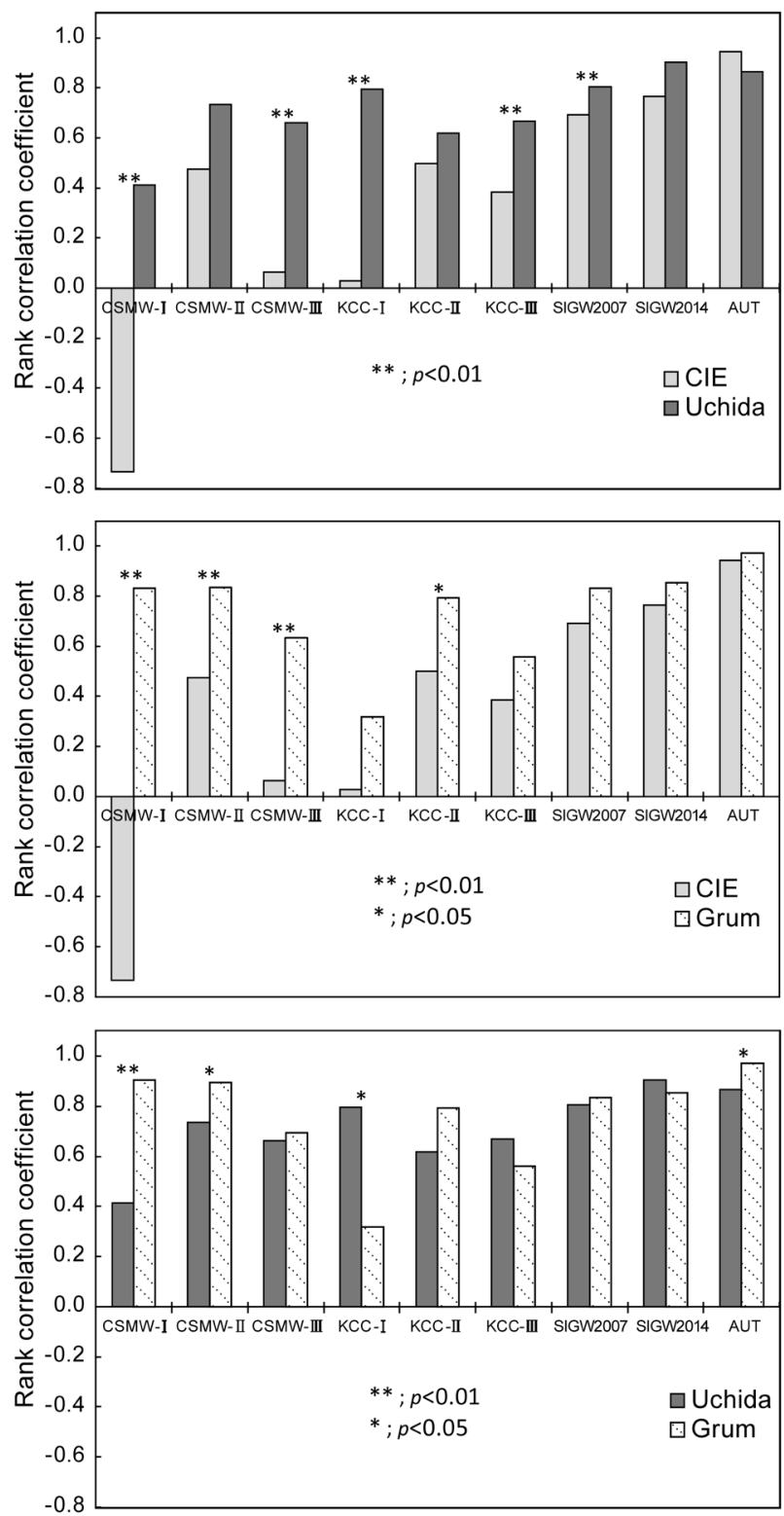

Figure 4 Comparisons of the predictive performance.

I, CSMW-II, CSMW-III, and SIGW-2007 data sets, the visual evaluation results do not show such a tendency.

The CIE whiteness formula is the sum of the $Y_{10}$ value and the value of $\left[800\left(x_{n, 10}-x_{10}\right)+1700\left(y_{n, 10}-y_{10}\right)\right]$, which correlates with the bluish purity of the sample. We analyzed what values they showed against the sample for each data set. The results are shown in Figure 7. In Figure 7, the abscissa represents the $x_{10}$ value, and the ordinate represents the $Y_{10}$ value and the value of $\left[800\left(x_{\mathrm{n}, 10}-x_{10}\right)+1700\left(y_{\mathrm{n}, 10}-y_{10}\right)\right]$, respectively. The filled circles in the figure indicate $Y_{10}$ values, and the open squares indicate the values of $\left[800\left(x_{n, 10}-x_{10}\right)+1700\right.$ $\left.\left(y_{n, 10}-y_{10}\right)\right]$. When we refer to Figure 7 , although the case for KCC-I is indistinct, we can see that the value of $\left[800\left(x_{\mathrm{n}, 10}-x_{10}\right)+1700\left(y_{\mathrm{n}, 10}-y_{10}\right)\right]$ monotonically increases as the $x_{10}$ value decreases, and the changes are considerably larger than the changes of the $Y_{10}$ value. That is,
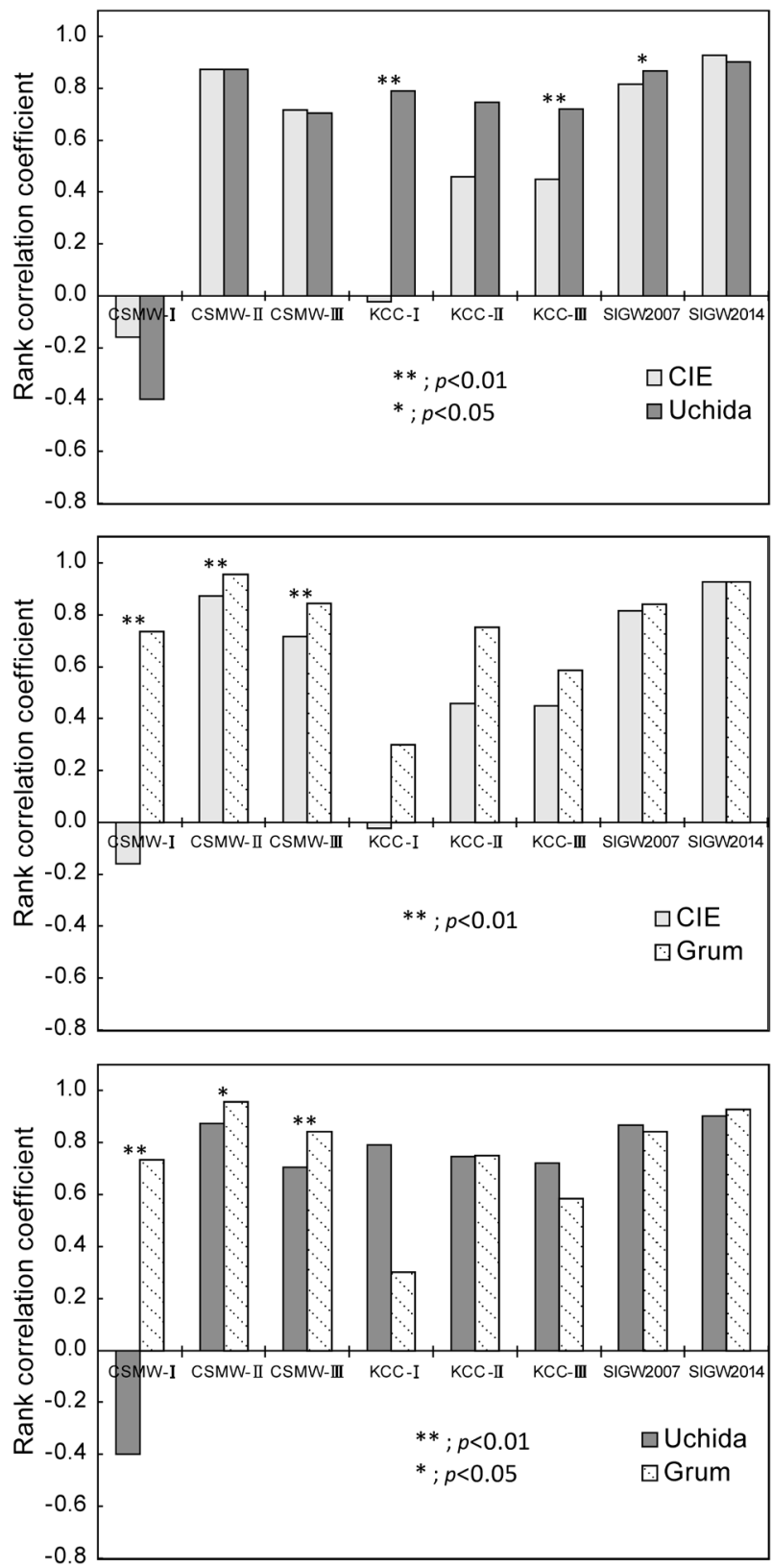

Figure 5 Comparisons of the predictive performance (samples outside the scope of the CIE whiteness formula excluded).

in most cases, the CIE whiteness index is determined by the bluish purity of the sample. Although excessive bluish tints reduce the perceived whiteness, unlike the Uchida or Grum whiteness formula, the CIE whiteness formula does not incorporate a penalty term that reduces whiteness for excessive tints; thus, a high whiteness value can be simply predicted for a sample with high bluish purity regardless of a change of the $Y_{10}$ value. It is believed that the low correlation with the visual evaluation results is due to the fundamental structure of the CIE whiteness formula, which does not have an appropriate penalty term. 

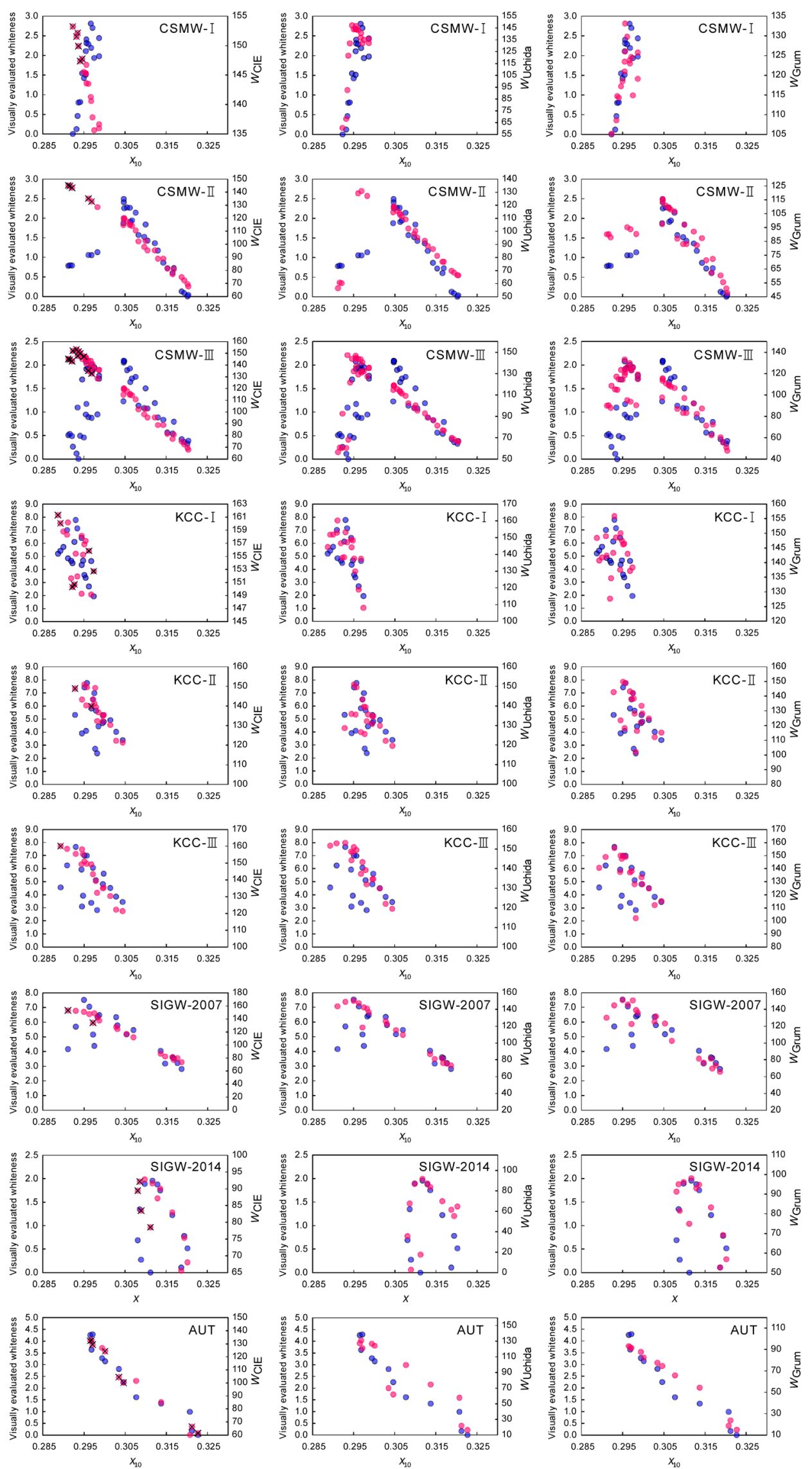

Figure 6 Visual evaluation results and the predicted values. The blue dots indicate the visual evaluation results and the red dots indicate the predicted values. 

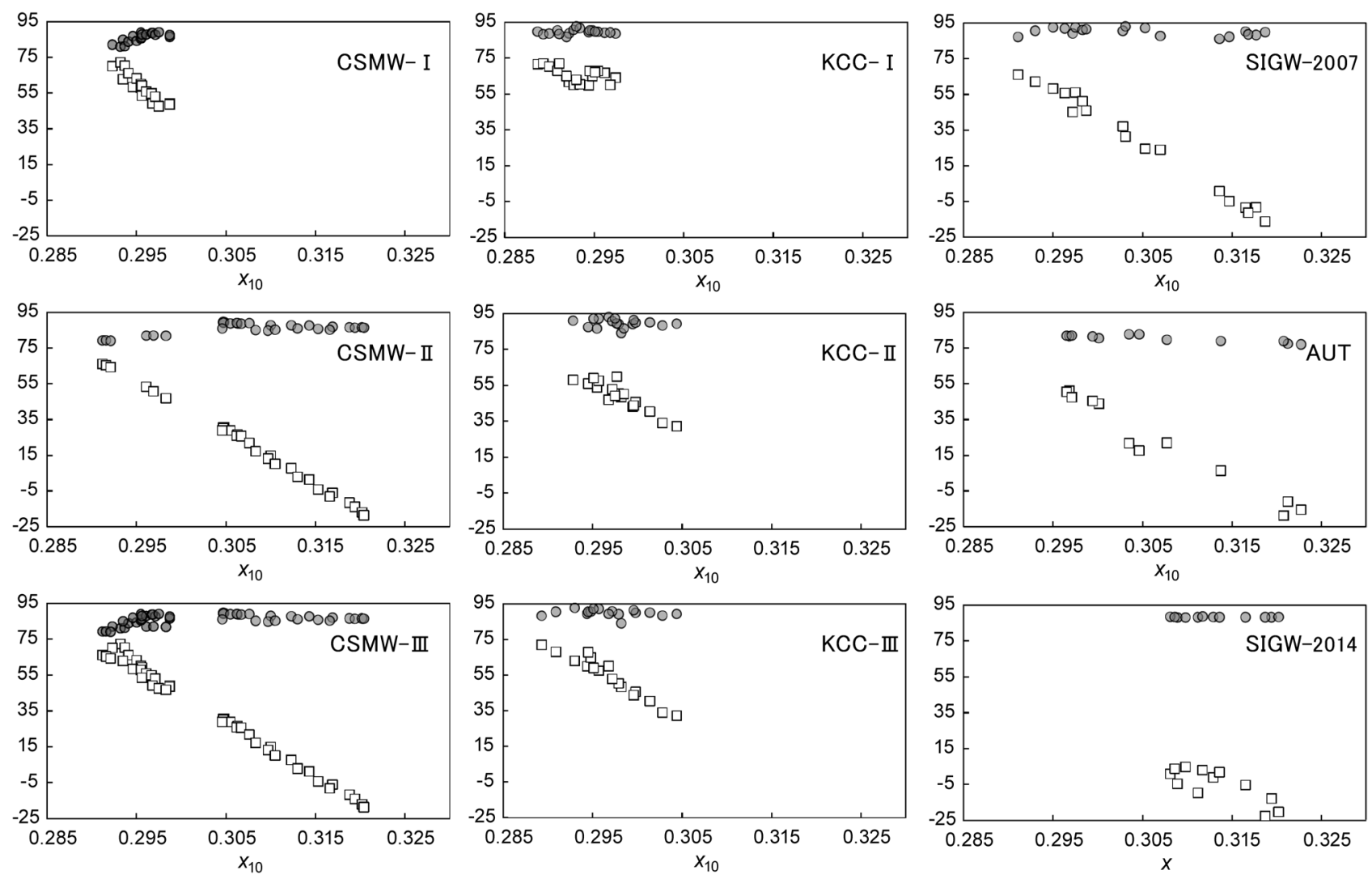

Figure 7 Changes of $Y_{10}$ value and the value of $\left[800\left(x_{n, 10}-x_{10}\right)+1700\left(y_{n, 10}-y_{10}\right)\right]$ against the $x_{10}$ value. The filled circles indicate $Y_{10}$ values, and the open squares indicate the values of $\left[800\left(x_{n, 10}-x_{10}\right)+1700\left(y_{n, 10}-y_{10}\right)\right]$.

\section{Conclusions}

We conducted comparisons of the predictive performance of 19 whiteness formulas using nine data sets. Results showed that the predictive performance of the Uchida and Grum whiteness formulas was significantly superior to that of the CIE whiteness formula. The predictive performance of the CIE whiteness formula was ranked 11th among the 19 whiteness formulas. It is believed that this low predictive performance is due to the structure of the CIE whiteness formula, which does not have a penalty term. Furthermore, it is found that although the structure of the Grum whiteness formula is simpler than that of the Uchida whiteness formula, it has better predictive performance.

\section{References}

(1) MacAdam, D. L.: The specification of whiteness, J. Opt. Soc. Am., 24-7, pp. 188-191 (1934).

(2) Judd, D. B.: A method for determining whiteness of paper, Pap. Trade J., 100, pp. 40-42 (1935).

(3) Wyszecki, G. and Stiles, W. S.: Color Science, 2nd Edition, John Wiley \& Sons, pp.837-839 (1982).

(4) Uchida, H. and Fukuda, T.: A history of establishing the CIE whiteness formula., J. Color Sci. Assoc. Jpn., 12-2, pp. 122-127 (1988) (in Japanese).

(5) CIE: Colorimetry 2nd Edition, CIE No. 15.2 (1986).
(6) CSMW: Technical Report, (1988) (in Japanese).

(7) JIS Z 8715: Colour specification-Whiteness of near white opaque materials (1991).

(8) CIE TC 1-95: The validity of the CIE whiteness and tint equations, http://www.cie.co.at/ technicalcommittees/validity-cie-whiteness-andtint-equations, (Accessed Aug. 30, 2018).

(9) Katayama, I.: Performance comparison of various whiteness formulas based on visual evaluation experiments, Proceedings of 28th CIE Session 2015, pp. 1072-1080 (2015).

(10) Katayama, I. and Uchida, H.: Whiteness based on the total spectral radiance factor measured under D65 fluorescent lamp, J. Color Sci. Assoc. Jpn., 383, pp. 188-189 (2014) (in Japanese).

(11) Katayama, I., Uchida, H., Sobagaki, H. and Baba, G.: Comparison of experimental results of whiteness evaluation by observers with different cultural background., J. Color Sci. Assoc. Jpn., 35-1, pp. 2-9 (2011) (in Japanese).

(12) Katayama, I., Iga, K., Isawa, S. and Suzuki, T.: Relationship between perceived whiteness and color vision characteristics, AIC2015 Conference Proceedings, pp. 782-786 (2015).

(13) Jafari, R. and Amirshahi, S. H.: A comparison of the CIE and Uchida whiteness formulas as predictor of average visual whiteness evaluation of tex- 
tiles, Text. Res. J., 77-10, pp. 756-763 (2007).

(14) Baba, G.: Quantitative evaluation of whiteness, Jpn. J. Optics, 10-1, pp. 11-17 (1981) (in Japanese).

(15) Katayama, I., Uchida, H., Sobagaki, H. and Baba, G.: Comparison of various whiteness formulae based on results of whiteness evaluation experiments, AIC2012 Conference Proceedings, pp. 610-613 (2012).

(16) CIE: Colorimetry 3rd Edition, CIE 15:2004 (2004).

(17) Uchida, H. and Fukuda, T.: Estimation of whiteness of fluorescent whitened cloths, J. Color Sci. Assoc. Jpn., 11-2, pp. 113-120 (1987) (in Japanese).

(18) Hunter, R. S.: New reflectometer and its use for whiteness measurement, J. Opt. Soc. Am., 50-1, pp. 44-48 (1960).

(19) Stensby, P. S.: Optical brightness and their evaluation III, Visual and instrumental evaluation of whiteness, Soap Chem. Specialties, 43-7, pp. 80-88 (1967).

(20) Fukuda, T. and Sugiyama, Y.: The evaluation of the whiteness of samples whitened by fluorescence, Acta Chromatica, 1-1, pp. 12-18
(1962).

(21) Croes, A. W.: Simple formula for calculating whiteness from photoelectric tristimulus data, J. Opt. Soc. Am., 49-8, pp. 830-831 (1959).

(22) Uchida, H.: Handbook of Color Science, 3rd Edition, University of Tokyo Ppess, pp. 593-598 (2011) (in Japanese).

(23) Grum, F., Witzel, R. F. and Stensby, P.: Evaluation of whiteness., J. Opt. Soc. Am., 64-2, pp. 210-215 (1974).

(24) Ganz, E.: Whiteness: Photometric specification and colorimetric evaluation, Appl. Opt., 15-9, pp. 20392058 (1976).

(25) Uchida, H.: A new whiteness formula, Color Res. Appl., 23-4, pp. 202-209 (1998).

(26) Ma, S., Wei, M., Liang, J., Wang, B., Chen, Y., Pointer, M. and Luo, M. R.: Evaluation of whiteness metrics, Light. Res. Technol., 50-3, pp. 429-445 (2018).

Part of this work was presented at 28th CIE Session, June 2015, Manchester, United Kingdom. 